\title{
Cefsulodin chocolate blood agar: a selective medium for the recovery of Haemophilus influenzae from the respiratory secretions of patients with cystic fibrosis
}

\author{
A. SMITH and M. BAKER \\ Public Health Laboratory and Division of Microbiology and Infectious Diseases, Queen's Medical Centre, \\ University Hospital, Nottingham NG7 $2 U H$
}

\begin{abstract}
A modified chocolate blood agar medium incorporating cefsulodin, a semi-synthetic cephalosporin, was developed and compared with non-selective chocolate blood agar and selective haemin-bacitracin blood agar for the routine isolation of Haemophilus influenzae from the respiratory secretions of patients with cystic fibrosis. The results showed that cefsulodin chocolate blood agar improved the recovery rate of $H$. influenzae in this group of patients. The medium was stable on storage for 10 days at $4^{\circ} \mathrm{C}$.
\end{abstract}

\section{Introduction}

Haemophilus influenzae is an important pathogen in patients with cystic fibrosis (CF), but may be difficult to detect in cultures of respiratory secretions because of overgrowth with Pseudomonas aeruginosa and Staphylococcus aureus. Cefsulodin is a semi-synthetic cephalosporin that has potent antibacterial activity against $P$. aeruginosa and some gram-positive bacteria including $S$. aureus but little effect against $H$. influenzae and many other gram-negative rods [1]. Thus the spectrum of antibacterial activity of cefsulodin is ideally suited for incorporation into selective media for the isolation of $H$. influenzae from the respiratory secretions of patients with CF. Other selective media for $H$. influenzae have used haemin and bacitracin [2], or $\mathrm{N}$ acetyl-D-glucosamine [3] as selective agents for haemophilus, but rely on anaerobic incubation to suppress the growth of $P$. aeruginosa. The application of cefsulodin disks to the surface of culture plates has been used to create an antibiotic gradient to avoid the suppression of more sensitive strains of $H$. influenzae while inhibiting $P$. aeruginosa [3].

The present study established the optimal concentration of cefsulodin for use in a selective medium for the isolation of $H$. influenzae from respiratory secretions of patients with CF. The effects of storage on stability of the medium were investigated and a trial of the new medium in comparison with non-

Received 11 Dec. 1996; revised version accepted 5 March 1997.

Corresponding author: Mr M. Baker. selective chocolate blood agar and haemin-bacitracin blood agar in a dilution technique was undertaken.

\section{Materials and methods}

\section{Preparation of media}

Chocolate blood agar was prepared by soaking $8 \mathrm{~g}$ of Blood Agar Base No. 2 (CM27; Oxoid) in $200 \mathrm{ml}$ of distilled water and autoclaving at $121^{\circ} \mathrm{C}$ for $15 \mathrm{~min}$. After cooling to $50^{\circ} \mathrm{C}, 14 \mathrm{ml}$ of defibrinated horse blood were added and the mixture was heated at $75^{\circ} \mathrm{C}$ in a water-bath until chocolating had taken place. After cooling again to $50^{\circ} \mathrm{C}$ cefsulodin (sodium salt; Sigma) was added as required and the media distributed in 19$\mathrm{ml}$ volumes. Haemin-bacitracin blood agar was prepared according to the formulation of Roberts and Cole [2].

Determination of MICs and selection of in-use concentration

Chocolate blood agar plates containing cefsulodin at final concentrations covering the range $1-128 \mathrm{mg} / \mathrm{L}$ were prepared and dried before use. Isolates of $H$. influenzae (18) and P. aeruginosa (18) isolated from the respiratory secretions of CF patients over a period of 3 weeks and subcultured daily on to chocolate blood agar and blood agar, respectively, were inoculated into $4.5-\mathrm{ml}$ volumes of brain-heart infusion broth and incubated at $37^{\circ} \mathrm{C}$ for $4-5 \mathrm{~h}$ in air enriched with $\mathrm{CO}_{2}$ 5\%. Escherichia coli NCTC 10418 and S. aureus NCTC 6571 were included as control organisms. After incubation the $P$. aeruginosa and $H$. influenzae cultures 
were diluted 1 in 10 (corresponding to $c \cdot 10^{7} \mathrm{cfu} / \mathrm{ml}$ and $c .5 \times 10^{5} \mathrm{cfu} / \mathrm{ml}$, respectively) with brain-heart infusion broth and $0.01 \mathrm{ml}$ was inoculated on to the cefsulodin chocolate blood agar plates with a multipoint inoculator. Dilutions of $H$. influenzae ( 1 in 1000) - corresponding to $c .5 \times 10^{3} \mathrm{cfu} / \mathrm{ml}-$ and the NCTC control organisms ( 1 in 10 and 1 in 1000) corresponding to $c .10^{7}$ and $c .10^{5} \mathrm{cfu} / \mathrm{ml}$, respectively - were prepared and inoculated in the same way. The plates were incubated for $18 \mathrm{~h}$ at $37^{\circ} \mathrm{C}$ in air enriched with $\mathrm{CO}_{2} 5 \%$, and the minimum inhibitory concentration (MIC) of cefsulodin for each strain was determined.

\section{Effects of storage}

MICs of cefsulodin for nine of the strains of $P$. aeruginosa and nine of the strains of $H$. influenzae, together with the $E$. coli and $S$. aureus controls, were also determined on plates that had been stored at $4^{\circ} \mathrm{C}$ for 10 days.

\section{Comparative clinical evaluation}

Sputa $(n=41)$, tracheal aspirates $(n=2)$ and cough swabs $(n=30)$ from patients with CF were cultured in parallel over a 9-week period on non-selective chocolate blood agar, cefsulodin chocolate blood agar and haemin-bacitracin blood agar. The sputa and tracheal aspirates were homogenised with an equal volume of Sputagest (Mast Diagnostics), inoculated on both selective media with sterile swabs and streaked around the plates with a sterile loop. A 1 in 10000 dilution of homogenised specimen in nutrient broth (previously shown to give discrete colonies) was then inoculated and streaked on non-selective chocolate blood agar in the same way. Cough swabs were inoculated directly on all three media (chocolate blood agar, haemin-bacitracin blood agar then cefsulodin chocolate blood agar) and streaked with a sterile loop. All manipulations were performed by a single operator. The non-selective chocolate blood agar and cefsulodin blood agar plates were incubated for $18 \mathrm{~h}$ at $37^{\circ} \mathrm{C}$ in air enriched with $\mathrm{CO}_{2} 5 \%$. The haemin-bacitracin blood agar plates were incubated anaerobically for $18 \mathrm{~h}$ at $37^{\circ} \mathrm{C}$. The identity of isolates of $H$. influenzae was confirmed by $\mathrm{X}$ and $\mathrm{V}$ factor dependence in subculture on nutrient agar (Blood Agar Base; Oxoid) with single and combined $\mathrm{X}$ and $\mathrm{V}$ factor disks (Oxoid). $P$. aeruginosa and $S$. aureus isolates were identified by standard tests [4].

\section{Statistical analysis}

Analysis of isolation rates was performed with Epi-Info 5 (Statcalc) and the $\chi^{2}$ distribution was calculated. Comparative results were considered as significant if the $\mathrm{p}$ value was $<0.05$.

\section{Results}

\section{Determination of MICs and selection of in-use concentration}

Eleven of the 18 strains of $P$ aeruginosa were inhibited by cefsulodin at a concentration of $8 \mathrm{mg} / \mathrm{L}$ (Table 1 ). In contrast, the MIC of cefsulodin for all the $H$. influenzae strains was $\geqslant 16 \mathrm{mg} / \mathrm{L}$ (low inoculum), or $\geqslant 32 \mathrm{mg} / \mathrm{L}$ (high inoculum) (Table 1). The MIC of cefsulodin for the control organisms was $32 \mathrm{mg} / \mathrm{L}$ ( $E$. coli) and $4 \mathrm{mg} / \mathrm{L}$ ( $S$. aureus) at both inoculum levels. On the basis of these results, cefsulodin $8 \mathrm{mg} / \mathrm{L}$ was chosen as the working concentration for incorporation into chocolate blood agar medium for use in the clinical evaluation exercise.

\section{Effect of storage}

MICs of cefsulodin for nine strains of $P$. aeruginosa and nine strains of $H$. influenzae were unchanged when tested on cefsulodin chocolate blood agar that had been stored at $4^{\circ} \mathrm{C}$ for 10 days.

\section{Comparative clinical evaluation}

Of the 73 specimens examined, the yield of the three main pathogens on any medium was: $P$. aeruginosa 17 , $S$. aureus 13 and $H$. influenzae 13 . Two specimens also yielded coliform bacilli.

There was no instance in which $H$. influenzae grew on non-selective media and not on selective media, and only one occasion in which growth was detected on all three media. In all instances in which there was growth of $H$. influenzae on haemin-bacitracin blood agar, there was enhanced growth on cefsulodin-chocolate blood agar, and on three occasions growth was detected on the cefsulodin-chocolate blood agar alone. In comparison with non-selective chocolate blood agar, the use of haemin-bacitracin blood agar increased the isolation rate of $H$. influenzae by $9.6 \%$. This increased rate was further improved to $17.8 \%$ with the use of cefsulodinchocolate blood agar (Table 2).

Table 1. MICs of cefsulodin for $P$ aeruginosa and $H$. influenzae

\begin{tabular}{|c|c|c|c|c|c|c|c|c|c|c|}
\hline \multirow[b]{2}{*}{ Organism } & \multirow{2}{*}{$\begin{array}{l}\text { Dilution of } \\
\text { inoculum }\end{array}$} & \multirow{2}{*}{$\begin{array}{l}\text { Inoculum size } \\
(\mathrm{cfu} / \mathrm{ml})\end{array}$} & \multicolumn{8}{|c|}{ Number of strains for which the MIC cefsulodin $(\mathrm{mg} / \mathrm{L})$ was } \\
\hline & & & 1 & 2 & 4 & 8 & 16 & 32 & 64 & 128 \\
\hline P. aeruginosa & 1 in 10 & $10^{7}$ & & 1 & 4 & 6 & 4 & 3 & & \\
\hline H. influenzae & 1 in 10 & $5 \times 10^{5}$ & & & & & & 2 & 4 & 12 \\
\hline H. influenzae & 1 in 1000 & $5 \times 10^{3}$ & & & & & 2 & 6 & 10 & \\
\hline
\end{tabular}


Table 2. Isolation rates of $H$. influenzae from 73 clinical specimens on non-selective and selective media

\begin{tabular}{lcc}
\hline & \multicolumn{2}{c}{$H$. influenzae } \\
\cline { 2 - 3 } Medium & $\begin{array}{l}\text { Number of } \\
\text { isolates }\end{array}$ & $\begin{array}{c}\text { Isolation } \\
\text { rate (\%) }\end{array}$ \\
\hline Chocolate blood agar & 3 & 4.1 \\
Cefsulodin-chocolate blood agar & 13 & 17.8 \\
Haemin-bacitracin blood agar & 10 & 13.7 \\
\hline
\end{tabular}

Isolation rates on the three media showed a significant difference $(p=0.03)$. The difference between chocolate blood agar and cefsulodin-chocolate blood agar was also significant (Yates-corrected p value of 0.02 ; 95\% confidence interval 1.29-28.66). Paired comparisons between chocolate blood agar and haeminbacitracin blood agar $(\mathrm{p}=0.08)$ and between cefsulodin-chocolate blood agar and haemin-bacitracin blood agar $(p=0.65)$ were not significant.

\section{Discussion}

H. influenzae commonly colonises the upper respiratory tract of healthy human subjects and caution must be observed in the interpretation of isolation of this organism from the respiratory secretions of patients suspected of lower tract infections. The use of dilution techniques for culture are well established as aids to diagnosis in situations in which overgrowth of oral flora may cause problems $[5,6]$.

In patients with chronic suppurative respiratory infection selective media have proved useful in the suppression of $P$. aeruginosa, particularly if cultures are incubated under anaerobic conditions [2]. The use of selective media for the isolation of $H$. influenzae from patients with $\mathrm{CF}$ ideally requires the incorporation of selective agents active against both $P$. aeruginosa and $S$. aureus as these organisms are commonly present in respiratory secretions from such patients and may obscure the more slowly growing $H$. influenzae. Cefsulodin provides the ideal spectrum of activity for this purpose and the results of the present study indicate that the incorporation of cefsulodin $8 \mathrm{mg} / \mathrm{L}$ into chocolate blood agar results in isolation rates at least as good as those obtained with haeminbacitracin blood-based media, without the need for anaerobic incubation.

We thank the staff of Nottingham Public Health Laboratory and Professor D. Greenwood for their help and encouragement.

\section{References}

1. Tsuchiya K, Kondo M, Nagatomo H. SCE-129, antipseudomonal cephalosporin: in vitro and in vivo antibacterial activities. Antimicrob Agents Chemother 1978; 13: 137-145.

2. Roberts D, Cole P. Use of selective media in bacteriological investigation of patients with chronic suppurative respiratory infection. Lancet 1980; 1: 796-797.

3. Möller LVM, van Alphen L, Grasselier H, Dankert J. N-AcetylD-glucosamine medium improves recovery of Haemophilius influenzae from sputa of patients with cystic fibrosis. J Clin Microbiol 1993; 31: 1952-1954.

4. Barrow GI, Feltham RKA (eds). Cowan and Steel's Manual for identification of medical bacteria, 3rd edn. Cambridge, Cambridge University Press. 1993.

5. Dixon JMS, Miller DC. Value of dilute inocula in cultura examination of sputum. Lancet $1965 ; 2 ; 1046-1048$.

6. Wilson MJB, Martin DE. Quantitative sputum culture as a means of excluding false positive reports in the routine microbiology laboratory. J Clin Pathol 1972; 25: 697-700. 